\title{
The UK Breast Cancer in Pregnancy (UKBCiP) Study. Incidence, diagnosis, management and short-term outcomes of breast cancer first diagnosed during pregnancy in the United Kingdom: A population-based descriptive study.
}

\author{
Claudia Hardy ${ }^{1}$, Julie Jones ${ }^{1}$, Andrew Brand ${ }^{2}$, Marian Knight ${ }^{3}$, and Philip Banfield ${ }^{1}$ \\ ${ }^{1}$ Glan Clwyd Hospital \\ ${ }^{2}$ Bangor University \\ ${ }^{3}$ National Perinatal Epidemiology Unit
}

June 22, 2020

\begin{abstract}
Objectives: To estimate the incidence of breast cancer diagnosed during pregnancy in the UK, to describe its management and short-term outcomes for mothers and babies. Design: A population-based descriptive study using the UK Obstetric Surveillance System (UKOSS). Setting: All UK consultant-led maternity units. Participants: All cases of breast cancer diagnosed first during pregnancy, between 1st October 2015 and 30th September 2017, with 84 confirmed cases analysed. Women with breast cancer diagnosed before pregnancy or with a recurrence were excluded. Method: Prospective case identification through monthly UKOSS mailings. Main outcome measures: Incidence of breast cancer arising for the first time in pregnancy, maternal mortality, severe maternal morbidity, perinatal mortality and severe neonatal morbidity. Results: The incidence found was 5.4/100,000 maternities (95\% CI 4.37, 6.70). Nine women (11\%) had an IVF pregnancy. During pregnancy, 30 women (36\%) underwent surgery and 37 women (44\%) received chemotherapy. Three women had major maternal morbidity during pregnancy. Two women died and there were two perinatal deaths. Conclusions: The incidence of breast cancer arising in pregnancy in the UK is similar to that reported in other countries. The higher proportion of IVF pregnancies among these women diagnosed with breast cancer during pregnancy needs further investigation. With caveats, the management followed that outside pregnancy, but there was considerable variation in practice. Although the short-term outcome was in general good for mothers and babies, a larger prospective study is required. It is often possible to avoid exposing the baby to iatrogenic prematurity.
\end{abstract}

\section{Hosted file}

UKBCiP BJOG V6.pdf available at https://authorea.com/users/334893/articles/460857-the-ukbreast-cancer-in-pregnancy-ukbcip-study-incidence-diagnosis-management-and-short-termoutcomes-of-breast-cancer-first-diagnosed-during-pregnancy-in-the-united-kingdom-apopulation-based-descriptive-study 